\title{
SOBRE A SÍNTESE E OBTENÇÃO DO CARBETO DE MOLIBDÊNIO COM DOPAGEM DE COBALTO COM ATMOSFERA DE METANO EM LEITO FIXO
}

\author{
C.P.B. de ARAUJO ${ }^{1}$, C.P. de SOUZA ${ }^{1}$ \\ ${ }^{1}$ Universidade Federal do Rio Grande do Norte, Departamento de Engenharia Química \\ E-mail para contato: cpbaraujo@gmail.com
}

\begin{abstract}
RESUMO - A rota de síntese do carbeto de molibdênio $\left(\mathrm{Mo}_{2} \mathrm{C}\right)$ a partir do heptamolibdato de amônio baseada na tecnologia de Boudart proporcionou ao processamento deste material ganhos significativos no tocante ao gasto energético total em relação ao processo convencional através da redução da temperatura e tempo de reação. Atualmente, diante de leis ambientais mais rigorosas referentes ao processamento e refino de petróleo e derivados, a busca por novos materiais com propriedades catalíticas diferenciadas tem se intensificado. Para tanto, a procura tem se baseado na dopagem de carbetos nanoestruturados com metais interessantes visando aumento de atividade catalítica. O molibdênio vem sendo extensivamente estudado como catalisador para reações de hidrotratamento, como hidrodessulfurização, hidrodenitrogenação; oxidação parcial de metano, entre outras. No presente trabalho $\mathrm{Mo}_{2} \mathrm{C}$, com adição de $\mathrm{Co}$, foi produzido via reação gássólido em reator de leito fixo com atmosfera redutora $\left(\mathrm{H}_{2}\right)$ e carburizante $\left(\mathrm{CH}_{4}\right)$. $\mathrm{O}$ precursor foi caracterizado antes da reação por TG/DTA, DR-X e, após a reação, por DR-X, MEV, FR-X para avaliação da sua potencialidade como catalisador nesses processos.
\end{abstract}

\section{INTRODUÇÃO}

Carbetos e nitretos de metais de transição, particularmente de $\mathrm{Mo}, \mathrm{Nb}$, e W, são interessantes quando aplicados à catálise em reações de importância industrial como hidrogenação de correntes pesadas de petróleo, hidrodessulfurização, transesterificação de óleos vegetais, oxidação catalítica, produção de gás de síntese, hidrodesaromatização de compostos, entre outras (BOROVINSKAYA, IGNATIEVA, et al., 2012). Observa-se também seu emprego como reforço de matrizes metálicas, promovendo melhoramento de propriedades como dureza e resistência à oxidação, além de possuir efeito na condutividade elétrica, inércia química e resistência a altas temperaturas das ligas produzidas (DE MEDEIROS, 2002).

Quando do seu uso em catálise, é interessante que carbetos possuam elevada seletividade, atividade e área superficial, bem como superfície livre de poluentes como carbono polimerizado e oxigênio (GIRAUDON, DEVASSINE, et al., 2000). Nessas condições, elevadas áreas superficiais possibilitam o contato íntimo entre catalisador e reagentes, favorecendo o processo catalítico. 


\section{9 a 22 de outubro de 2014 \\ Florianópolis/SC}

Elevadas áreas superficiais também são fundamentais quando do emprego de carbetos em metais duros. Neste caso, quando associadas a diminutos tamanhos de partícula, permitem a obtenção de propriedades mecânicas mais interessantes que aquelas alcançadas quando da utilização de partículas de maior tamanho.

A larga tendência para produção de materiais de tamanho nanométrico tem impulsionado a produção de carbetos de metais de transição nesta faixa de tamanho para uso como ferramentas de corte, reforço de estruturas aeroespaciais, catalisadores heterogêneos, entre outros (BOROVINSKAYA, IGNATIEVA, et al., 2012).

Convencionalmente, a produção de carbetos deriva de processos metalúrgicos nos quais o metal é reagido com uma fonte de carbono, geralmente negro de fumo, a elevadas temperaturas $\left(>1200{ }^{\circ} \mathrm{C}\right.$ para o Mo). Tal processo resulta em materiais com baixa atividade catalítica, principalmente devido à suas baixas áreas superficiais, associadas a uma larga distribuição de tamanhos e presença de carbono ligado na superfície do sólido. A obtenção de materiais que atendam essa exigência foi, por muito tempo, um fator limitante para a aplicação de compostos de Mo e W em catálise (VOLPE e BOUDART, 1985). Através do desenvolvimento por Boudart et al. da metodologia de obtenção de carbetos via reação gás-sólido em 1985 pode-se obter WC com elevadas áreas superficiais. Oyama et. al (OYAMA, 1992) também desenvolveram estudos neste sentido produzindo outros carbetos monometálicos pela mesma técnica.

Atualmente, diante de leis ambientais mais rigorosas sobre o processamento e refino de petróleo e derivados, a busca por novos materiais com propriedades catalíticas diferenciadas para uso nas etapas de tratamento secundário tem se intensificado. Para tanto, a procura tem se baseado na dopagem de carbetos nanoestruturados com metais interessantes.

A dopagem de carbetos com outros metais possibilita incremento no poder catalítico desses, uma vez que a adição do metal envolve a modificação da estrutura cristalina, aumento de sítios catalíticos e possui efeito, também, sobre a atividade do produto final (CHENG e HUANG, 2010). $\mathrm{Co}_{3} \mathrm{Mo}_{3} \mathrm{C}$ é reportado como sendo mais ativo que o catalisador de ferro duplamente promovido utilizado para síntese de amônia pelo processo de Bosh-Haber (AL SHALWI, 2012), sendo um indicativo das possíveis aplicações de $\mathrm{Mo}_{2} \mathrm{C}$ com dopagens variadas desse metal.

No presente trabalho o carbeto de molibdênio dopado com cobalto foi sintetizado com duas percentagens de cobalto, $2,5 \%$ e $5 \%$ e, em seguida, caracterizado por FR-X, DR-X e MEV. O tamanho do cristalito foi determinado pelo método de Sherrer e Williamson-Hall mediante dados de difração de raios-X.

\section{MATERIAIS E MÉTODOS}

Heptamolibdato de amônio [(NH4) ${ }_{6} \mathrm{Mo}_{7} \mathrm{O}_{24} \cdot 4 \mathrm{H}_{2} \mathrm{O}$ ] (VETEC, $99 \%$ ) foi manualmente misturado em almofariz com nitrato de cobalto $\left[\mathrm{Co}\left(\mathrm{NO}_{3}\right)_{2} \cdot 6 \mathrm{H}_{2} \mathrm{O}\right]$ (CROMATO, 98\%) nas porcentagens de com 2,5 e $5 \%$ (Co/Mo).O produto dessa mistura física foi submetido a análise térmica e diferencial TG/DTA para avaliação do comportamento das fases ao longo do perfil de aquecimento, bem como 
da perda de massa. $\mathrm{O}$ material produzido foi submetido à reação de carbo-redução em reator de leito fixo, em seguida. Primeiramente, a atmosfera do reator foi purgada com fluxo de Argônio. Iniciou-se, então, o fluxo da mistura gasosa $\mathrm{CH}_{4}$ e $\mathrm{H}_{2}(95 \%)$ a volume total de $15 \mathrm{~L} / \mathrm{h}$. Taxas de aquecimento de $5{ }^{\circ} \mathrm{C} \cdot \mathrm{min}^{-1} \mathrm{com}$ temperaturas de reação de $700{ }^{\circ} \mathrm{C}$ e $750{ }^{\circ} \mathrm{C}$ e, patamar de 180 min foram utilizadas em cada caso. As amostras foram nomeadas de acordo com o percentual de cobalto (Co/Mo) e temperatura de patamar $\left(\mathrm{x} \%-\mathrm{T}^{\circ} \mathrm{C}\right)$.

Os produtos da reação foram acondicionados em recipiente apropriado imediatamente após atingirem temperatura ambiente e foram caracterizados por FR-X (EDX-720, atmosfera de ar), DR-X (SHIMADZU XRD- 6000, $\mathrm{Cu} \mathrm{K \alpha}$, operado a $30 \mathrm{kV}$ e $30 \mathrm{~mA}, 2^{\circ}{ }^{\circ} \mathrm{min}^{-1}$ ), MEV (Hitachi Tabletop Microscope TM-3000, aumentos de 3, 5 e 8 x 103).

\section{RESULTADOS E DISCUSSÃO}

\subsection{Avaliação do precursor}

\section{Análise Térmica (TG/DTA): A}

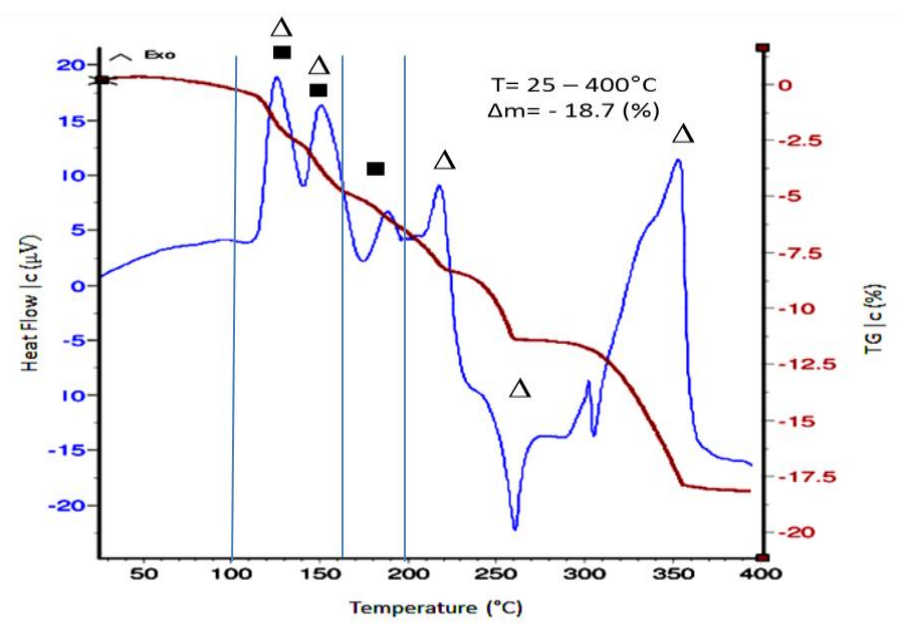

Figura 1 apresenta a análise térmica do precursor com 5\% de Co realizada em atmosfera oxidante estagnada. Pode ser percebida, ao longo do caminho térmico, a perda de massa suave, bem como eventos endo e extotérmicos, de magnitude expressiva. Quatro etapas de perda de massa podem ser majoritariamente atribuídas ao heptamolibdato de amônio $(\Delta)$ por comparação com dados da literatura disponível para este processo. Os demais eventos são associados ao nitrato de cobalto ( $\mathbf{\square})$. Pode-se perceber, por análise da figura, que as duas primeiras etapas de perda de massa são somente distinguíveis entre si através da análise da curva derivativa, a qual indica dois picos exotérmicos no intervalo de $100-160^{\circ} \mathrm{C}$. Estes eventos de perda de massa são relativos à evaporação de água de hidratação do precursor. Em seguida, se verifica a terceira etapa, a qual pode ser atribuída à liberação de compostos nitrogenados (NOx), ao qual está associado evento exotérmico do sistema. Nos eventos posteriores, compostos amoniacais são liberados juntamente com o restante de água ligada. 
Difração de Raios-X( DR-X): A análise por difração de raios-X do precursor calcinado à temperatura de $700^{\circ} \mathrm{C}$ em mufla, segue conforme a Figura 2. A identificação de fases realizada indicou a presença de fase única $\mathrm{MoO}_{3}$. Isso sugere que o Co adicionado encontra-se disperso na estrutura cristalina na forma de defeitos pontuais, não formando fase única.

\subsection{Avaliação dos produtos}

Fluorescência de Raios-X (FR-X): $\mathrm{O}$ teor de cobalto presente do produto final foi determinado por análise por fluorescência de raios-X. A Tabela 1 contempla estes dados. A Figura 3 apresenta a curva de fluorescência obtida, ilustrando intensidade e posição dos picos de interesse obtidos para todas as amostras (Mo- $\mathrm{L} \alpha-2.30 \mathrm{keV}, \mathrm{Co}-\mathrm{K} \alpha-6.94 \mathrm{keV}$, Co- $\mathrm{K} \beta-7.66 \mathrm{keV}$, Mo-K $\alpha-17.48 \mathrm{keV}$, Mo-K $\beta-19.64 \mathrm{keV})$. Como se pode perceber, existe concordância significativa entre os valores pretendidos e obtidos no produto final.

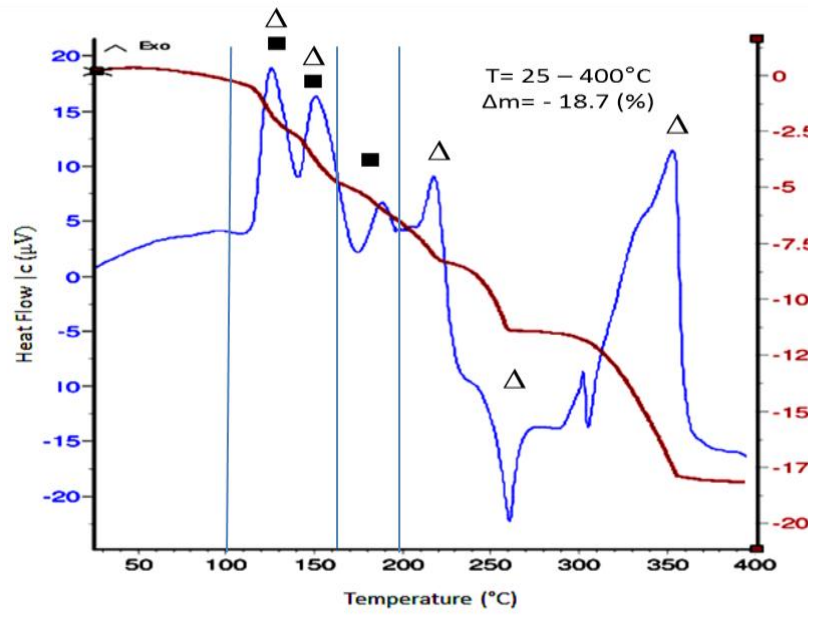

Figura 1: Análise termogravimétrica e diferencial para amostra de precursor. Atmosfera oxidante estagnada.

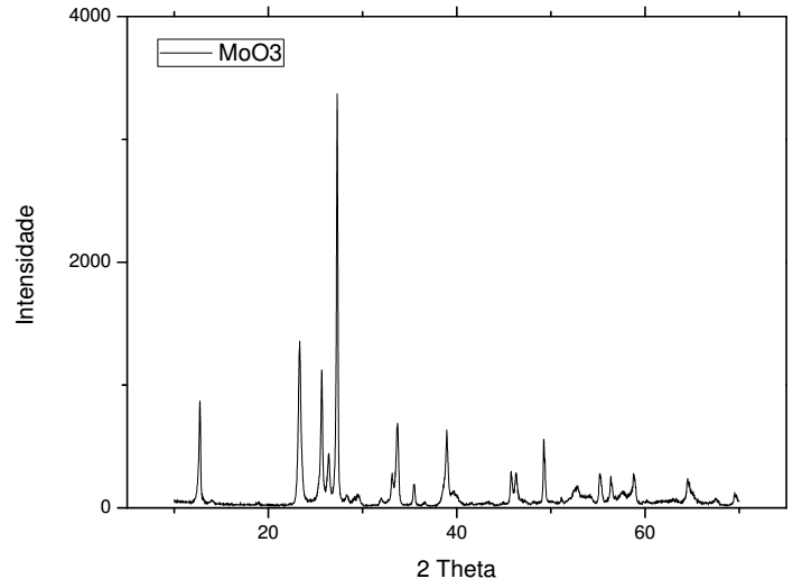

Figura 2: Padrão de difração obtido para o precursor da reação de carbo-redução após calcinação em forno mufla a $700^{\circ} \mathrm{C}$ 


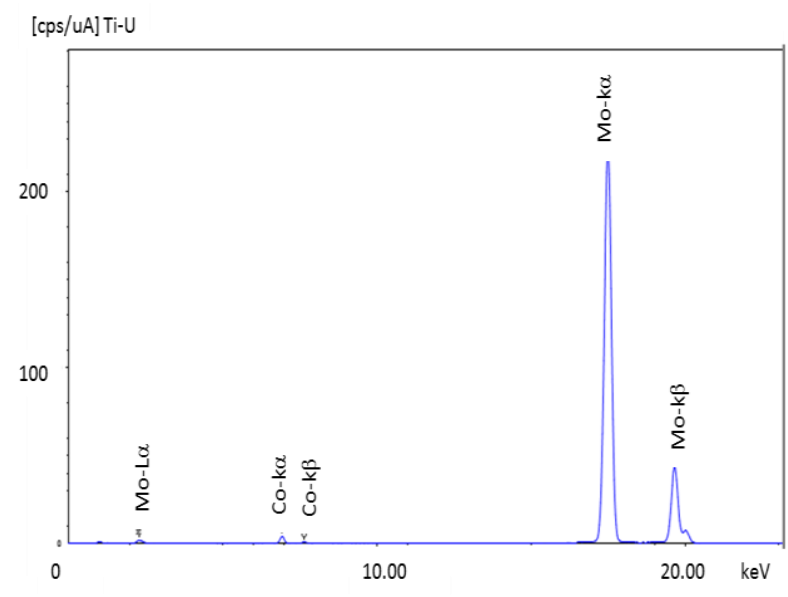

Tabela 1: Percentual de Co no produto final

(FR-X)

\begin{tabular}{|c|c|c|c|}
\hline Amostra & $\begin{array}{c}\text { \%peso } \\
\text { Co }\end{array}$ & $\begin{array}{c}\text { \%molar } \\
\text { Co }\end{array}$ & $\begin{array}{c}\text { Razão } \\
\text { Co/Mo }\end{array}$ \\
\hline $\begin{array}{c}2.5 \%- \\
700^{\circ} \mathrm{C}\end{array}$ & 1.349 & 2.178 & 0.0223 \\
\hline $\begin{array}{c}2.5 \%- \\
750^{\circ} \mathrm{C}\end{array}$ & 1.359 & 2.194 & 0.0224 \\
\hline $5 \%-700^{\circ} \mathrm{C}$ & 2.754 & 4.408 & 0.0461 \\
\hline $5 \%-750^{\circ} \mathrm{C}$ & 2.762 & 4.420 & 0.0462 \\
\hline
\end{tabular}

Figura 3: Gráfico ilustrativo da análise de fluorescência de raios-X do produto final da reação de carbo-redução

Difração de Raios-X( DR-X): A análise por difração de raios-X dos pós obtidos pode ser analisada na Figura 4. Mediante o aumento da temperatura de reação, o percentual da fase de carbeto aumentou em detrimento da composição de óxidos. Os materiais obtidos a $750^{\circ} \mathrm{C}$ formaram $\mathrm{Mo}_{2} \mathrm{C}$, em fase única, no qual o Co se apresenta somente de maneira dispersa. A $700^{\circ} \mathrm{C}$, duas fases podem ser verificadas, sendo elas $\mathrm{Mo}_{2} \mathrm{C}$ e $\mathrm{MoO}_{2}$. Não foi detectada, por este método, a presença de uma fase óxido combinando Mo e Co, devido, principalmente, à baixa concentração utilizada, porém, conforme verificado por FR-X, o dopante encontra-se presente no material produzido. 


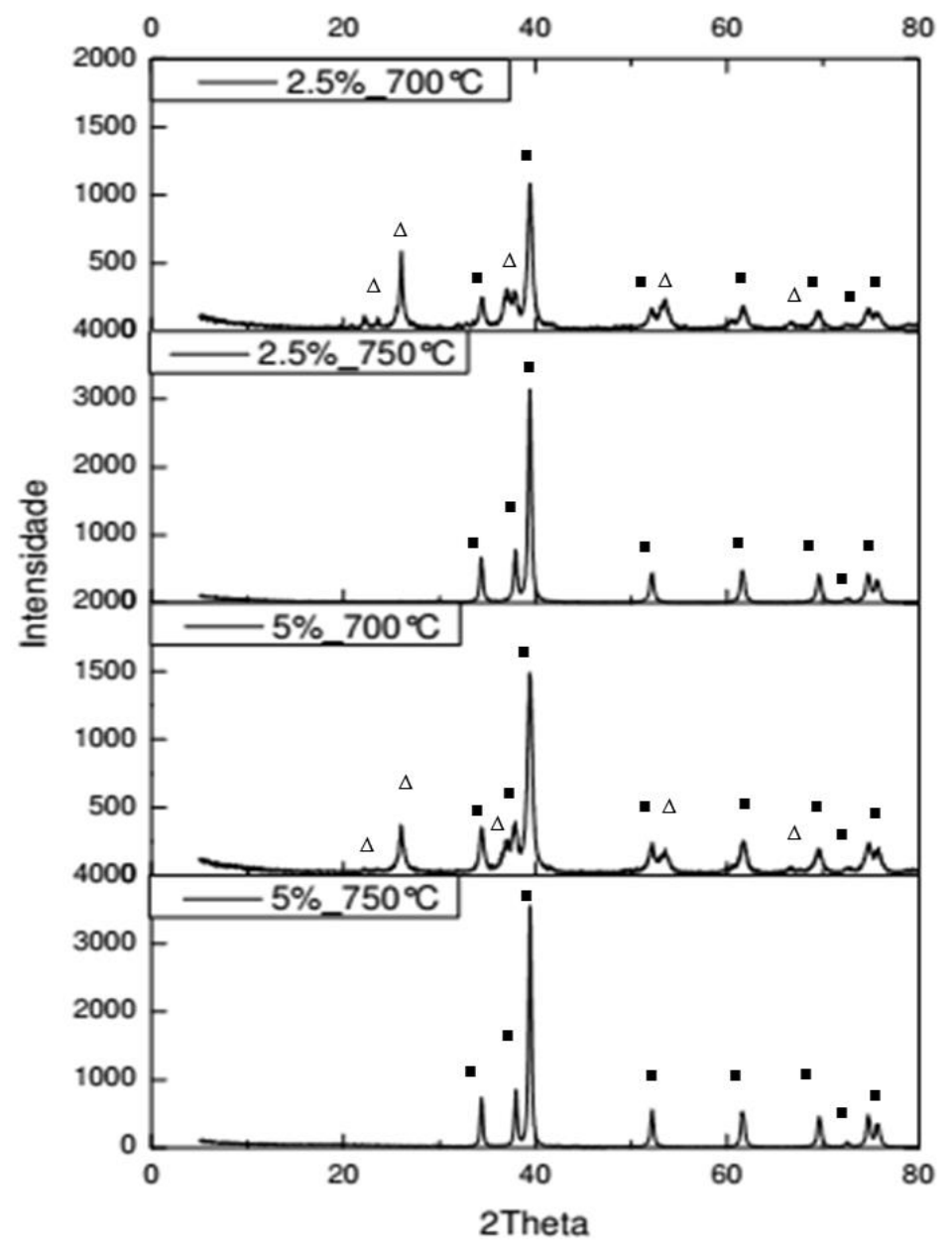

Figura 4: Difratograma de raios-X das amostras apresentando diferentes $\%$ Co e diferentes $\mathrm{T}$ de patamar. $\Delta$ indica os picos relativos à fase $\mathrm{MoO}_{2} \mathrm{e} \mathbf{\boldsymbol { m }}$ indica os picos relativos à fase $\mathrm{Mo}_{2} \mathrm{C}$.

Avaliação do tamanho de cristalito: A partir dos dados de DR-X foi possível estimar o tamanho de cristalito. Tal estimativa se baseou nos métodos de Scherrer e Williamson-Hall (WH). Os resultados obtidos podem ser observados na Tabela 2. Conforme esperado, devido ao fato de o método de W-H levar em consideração as microdeformações na estrutura cristalina, a contabilização resulta em tamanho de cristalito maior. Analisando os resultados obtidos para as mesmas temperaturas, percebe-se um aumento do tamanho de partícula com a elevação do teor de Co. Essa observação está de acordo com o esperado, uma vez que o raio atômico do Co é menor que o do Mo e deve ocupar sítios intersticiais na estrutura, promovendo distorções e aumento da distância interplanar, e consequentemente do cristalito. Em análise para os dados obtidos à com mesmo teor de Co, o aumento de temperatura promoveu um crescimento do cristalito. 
Tabela 2: Análise dos tamanhos de cristalito pelos métodos de W-H e Scherrrer.

\begin{tabular}{|c|c|c|c|}
\hline Amostra & W-Hall (nm) & Microdeformaçao W-Hall & Scherrer (nm) \\
\hline $2.5 \%-700^{\circ} \mathrm{C}$ & 30.82 & 0.002600 & 16.65 \\
\hline $2.5 \%-750^{\circ} \mathrm{C}$ & 28.04 & 0.000700 & 24.19 \\
\hline $5 \%-700^{\circ} \mathrm{C}$ & 46.39 & 0.011675 & 14.89 \\
\hline $5 \%-750^{\circ} \mathrm{C}$ & 34.22 & 0.000400 & 29.52 \\
\hline
\end{tabular}

Microscopia Eletrônica de Varredura (MEV): Os produtos obtidos após a carburização foram submetidos à microscopia eletrônica de varredura para analise qualitativa da distribuição do metal dopante no material, observação da morfologia e distribuição de tamanhos do produto. Para a amostra $2.5 \%-700{ }^{\circ} \mathrm{C}$, com aumento de $5 \mathrm{kx}$, pode-se observar claramente regiões com diferentes contrastes, indicando a distribuição do Co no material, de acordo com a Figura 5. Além disso, é notória a presença de regiões de aglomerados de partículas de tamanho aparentemente uniforme.

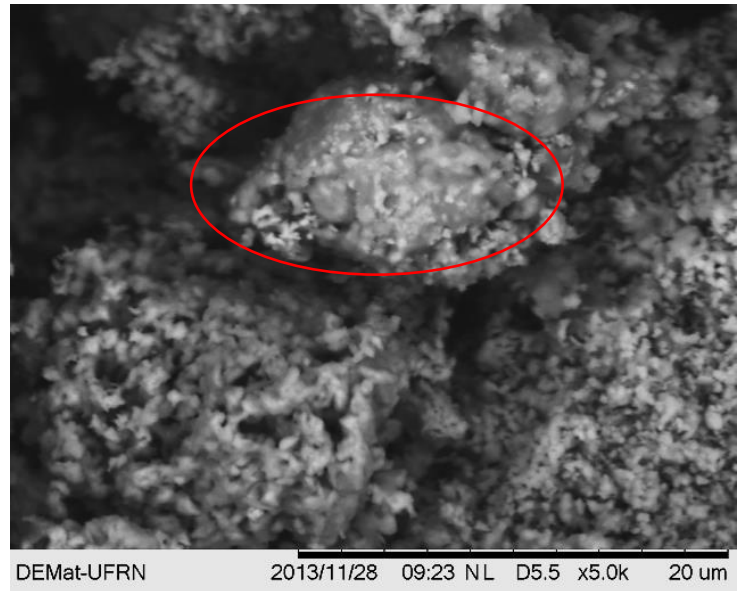

Figura 5: MEV da amostra 2.5\%$700^{\circ} \mathrm{C}$ com aumento de 5000x. Imagens obtidas com equipamento do DEMATUFRN

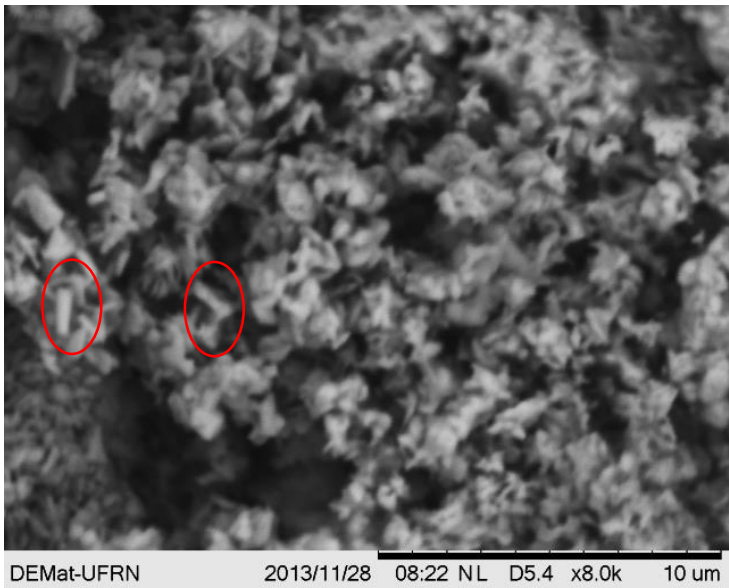

Figura 6: MEV da amostra 2.5\%$750^{\circ} \mathrm{C}$ com aumento de 8000x. Imagens obtidas com equipamento do DEMATUFRN.

A Figura 6 ilustra a amostra $2.5 \%-750{ }^{\circ} \mathrm{C}$ com aumento de 8000 x. Observam-se cristais de forma aparentemente hexagonal, conforme indicado nas regiões circuladas. Tal observação está de acordo com o obtido por Gomes (GOMES, 2006). Já a Figura 7, apresenta a micrografia do material obtido a $700{ }^{\circ} \mathrm{C}$ com $5 \%$ de $\mathrm{Co}$, sendo possível notar uma heterogeneidade do tamanho das partículas, a sua aglomeração, e, também, uma maior tendência à sinterização, em relação à Figura 5. Tal fenômeno pode ser devido ao aumento do \% de Co, diminuindo assim a temperatura necessária para a sinterização do material. A partir da Figura 8, a qual apresenta resultados obtidos com $5 \%$ de Co a $750{ }^{\circ} \mathrm{C}$, é possível observar uma tendência à maior 
aglomeração das partículas. Em todas as imagens foi possível perceber a presença do cobalto disperso de maneira razoavelmente uniforme, em confirmação com o obtido por FR-X (presença do metal) e DR-X (disperso sem formar fase única).

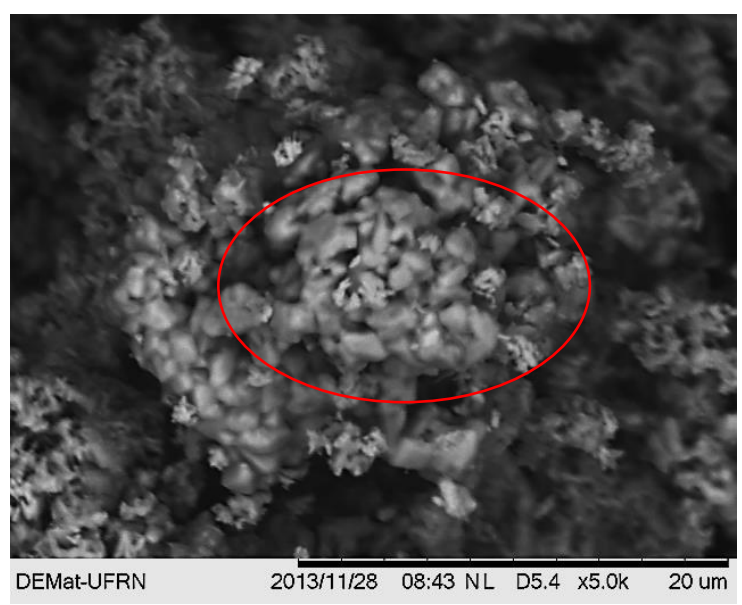

Figura 7: $\mathrm{MEV}$ da amostra $5 \%-700^{\circ} \mathrm{C}$ com aumento de 5000x. Imagens obtidas com equipamento do DEMAT-UFRN

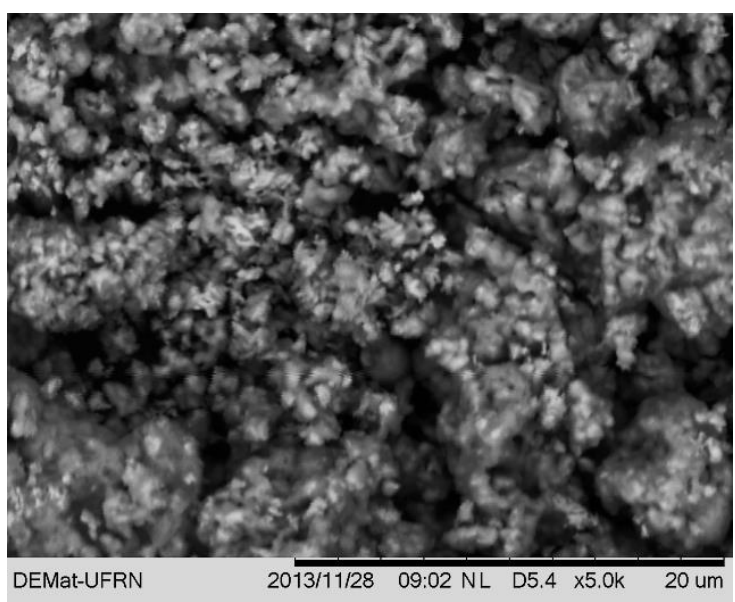

Figura 8: $\mathrm{MEV}$ da amostra $5 \%-750^{\circ} \mathrm{C}$ com aumento de 5000x. Imagens obtidas utilizando equipamento do DEMAT-UFRN

\section{CONCLUSÕES}

No presente trabalho foi possível obter carbeto de molibdênio dopado com cobalto à temperatura de $750^{\circ} \mathrm{C}$ mediante carburização de precursor $\left(\left[(\mathrm{NH} 4)_{6} \mathrm{Mo}_{7} \mathrm{O}_{24} \cdot 4 \mathrm{H}_{2} \mathrm{O}\right]+\right.$ $\left.\left[\mathrm{Co}\left(\mathrm{NO}_{3}\right)_{2} .6 \mathrm{H}_{2} \mathrm{O}\right]\right)$ em atmosfera redutora constituída de $\mathrm{CH}_{4}(5 \%)$ e $\mathrm{H}_{2}$, com vazão total de $15 \mathrm{~L} / \mathrm{h}$ e tempo de patamar de 180 minutos, em reator de leito fixo. Nessas condições é observado, por DR-X, a formação de fase única de $\mathrm{Mo}_{2} \mathrm{C}$ na qual se infere a dispersão do Co de maneira homogênea devido à confirmação por FR-X da sua presença no material. Tal material apresenta potencialidade para aplicação em reações catalíticas tanto na área das reações hidrotratamento de correntes de refino de petróleo, como o $\mathrm{Mo}_{2} \mathrm{C}$ já tem sido investigado, como em reações do tipo Fischer-Tropsch nas quais o cobalto é conhecidamente utilizado como promotor. Adicionalmente, devido ao seu tamanho de cristalito nanométrico o material teoricamente formaria fina película fluida envolvendo-o em um processo real, minimizando assim possíveis resistências físicas associadas ao processo de transferência de massa.

\section{REFERÊNCIAS}

AL SHALWI, M. The preparation of molybdenum carbonitrides by single source routes and a study of their lattice reactivity. Universidade de Glasgow, Escola de Química. Glasgow. 2012. 
BOROVINSKAYA, I. et al. Tungsten Carbide - Processing and Applications. 1a. ed. [S.l.]: INTECH, 2012.

CHENG, J.; HUANG, W. Effect of cobalt (nickel) content on the catalytic performance of molybdenum carbides in dry methane reforming. Fuel Processing Technology, v. 91, p. 185193, 2010.

DE MEDEIROS, F. F. P. Síntese de carbetos de tungstênio e nióbio a baixa temperatura através de reação gás-sólido em reator de leito fixo. Natal: [s.n.], 2002.

ELSEVIER. Refractory Materials: A series of Monographs. [S.l.]: [s.n.], v. 7, 1970.

GIRAUDON, J. M. et al. Synthesis of Tungsten Carbides by Temperature-Programmed Reaction with $\mathrm{CH} 4 / \mathrm{H} 2$ Mixtures.Influence of the $\mathrm{CH} 4$ and Hydrogen Content in the Carburizing Mixture. Journal of Solid State Chemistry, 154, 2000. 412-426.

GOMES, K. K. P. Sintese e caracterização do carbeto de molibdênio nanoestruturado para fins catalíticos na reação de oxidação parcial do metano. Universidade Federal do Rio Grande do Norte. Natal. 2006.

MEDEIROS, F. F. P. et al. Synthesis of tungsten carbide through gas - solid reaction at low temperatures. Materials Science and Engineering A315, 2001. 58-62.

OYAMA, S. T. Crystal structure and chemical reactivity of transition metal carbides and nitrides. Journal of solid state chemistry, v. 96, p. 442-445, 1992.

OYAMA, S. T. Preparation and catalytic properties of transition metal carbides and nitrides. Catalysis Today, v. 15, p. 179-200, 1992.

OYAMA, T. S.; KAPOOR, R. Transition metal carbides and nitrides, p. 156-159.

VOLPE, L.; BOUDART, M. Compounds of Molybdenum and Tungsten with High Specific Surface Area: II Carbide. Journal of Solid State Chemistry, v. 59, p. 348-356, 1985. 